\title{
Philosophiques
}

\section{Pluraliser - le legs de Heidegger}

\section{Maxime Plante}

Volume 47, numéro 2, automne 2020

\section{Derrida en cours}

URI : https://id.erudit.org/iderudit/1075128ar

DOI : https://doi.org/10.7202/1075128ar

Aller au sommaire du numéro

\section{Éditeur(s)}

Société de philosophie du Québec

\section{ISSN}

0316-2923 (imprimé)

1492-1391 (numérique)

Découvrir la revue

\section{Citer cet article}

Plante, M. (2020). Pluraliser — le legs de Heidegger. Philosophiques, 47(2),

351-368. https://doi.org/10.7202/1075128ar

\section{Résumé de l'article}

La publication récente de Geschlecht III permet pour la première fois d'embrasser de manière synoptique le projet Geschlecht et d'en apprécier la cohérence d'ensemble. Je prends le parti de lire la série Geschlecht comme la scène d'un héritage où Derrida se débat littéralement avec le legs heideggérien. J'isole en particulier le ressort de la critique du rassemblement (Versammlung) qui organise la lecture de Derrida dans toute la série. Je propose de distinguer deux niveaux à cette lecture : l'un, où est mis en scène le refus d'un héritage jugé métaphysique ou suspect ; l'autre, que l'on méconnaît plus volontiers, qui pour sa part met en scène un héritage partagé (don, promesse, économie). On verra la valeur de rassemblement apparaître tour à tour de l'un et de l'autre côté de ces lignes de partage, et pluraliser le legs devenir l'enjeu de la scène.
Ce document est protégé par la loi sur le droit d'auteur. L'utilisation des services d'Érudit (y compris la reproduction) est assujettie à sa politique d'utilisation que vous pouvez consulter en ligne.

https://apropos.erudit.org/fr/usagers/politique-dutilisation/ 


\title{
Pluraliser — le legs de Heidegger
}

\author{
MAXIME PLANTE \\ Chercheur postdoctoral \\ Département de philosophie et Département des littératures de langue française \\ Université de Montréal
}

\begin{abstract}
RÉSUMÉ. - La publication récente de Geschlecht III permet pour la première fois d'embrasser de manière synoptique le projet Geschlecht et d'en apprécier la cohérence d'ensemble. Je prends le parti de lire la série Geschlecht comme la scène d'un héritage où Derrida se débat littéralement avec le legs heideggérien. J'isole en particulier le ressort de la critique du rassemblement (Versammlung) qui organise la lecture de Derrida dans toute la série. Je propose de distinguer deux niveaux à cette lecture: I'un, où est mis en scène le refus d'un héritage jugé métaphysique ou suspect; l'autre, que l'on méconnaît plus volontiers, qui pour sa part met en scène un héritage partagé (don, promesse, économie). On verra la valeur de rassemblement apparaître tour à tour de l'un et de l'autre côté de ces lignes de partage, et pluraliser le legs devenir l'enjeu de la scène.
\end{abstract}

\begin{abstract}
The recent publication of Geschlecht III allows us, for the first time, to synoptically embrace the Geschlecht project and to appreciate its overarching consistency. I choose to read the Geschlecht series as the scene of an inheritance whereby Derrida wrestles with Heidegger's legacy. More specifically, I identify the linchpin of Derrida's critique of gathering (Versammlung), which organizes his reading throughout the series. I then distinguish between two layers within this reading: the first, wherein he stages the refusal of an inheritance deemed metaphysical or suspicious, and the less well-known second, which sets the stage for a shared heritage (gift, promise, economy). The value of gathering thus consecutively appears on one and the other side of these dividing lines, until the legacy's pluralization turns out to be what's at stake in the scene.
\end{abstract}

Nous ne nous débarrasserons jamais du corps de Heidegger. JaCQues DeRrida ${ }^{1}$

Heidegger [...]. C’est pour moi une sorte de veilleur, de pensée qui veille tout le temps sur moi.

JACQUes DeRrida ${ }^{2}$

Une fois de plus, il sera question du rapport entre Derrida et Heidegger. Une fois de plus. Comment ne pas réprimer un soupir devant la monotonie de cette question, battue et rebattue au point de devenir un lieu commun de la recherche, tellement qu'on n'y voit plus bien la fécondité d'y séjourner encore, une fois de plus. La lassitude n'est pas seulement nôtre, elle aura aussi été partagée par Derrida: "J'imagine l'impatience de certains [...]

1. Cite dans David Farrell Krell, Phantoms of the Other: Four Generations of Derrida's Geschlecht, Albany (NY), State University of New York Press, 2015, p. 130.

2. Cité dans Dominique Janicaud, Heidegger en France. II. Entretiens, Paris, Albin Michel, 2001, p. 103.

PHILOSOPHIQUES 47/2 - Automne 2020, p. 351-368 
devant le temps de ce séjour auprès de Heidegger. Encore Heidegger! Et ce retour de Heidegger et ce retour à Heidegger! Est-ce que cela ne suffit pas? Est-ce encore actuel ${ }^{3}$ ?"

Sur le rapport entre Derrida et Heidegger, tout aurait été dit et d'abord l'évidence: Derrida se trouverait en situation de rapport critique vis-à-vis de Heidegger. Cette caractérisation n'est bien entendu pas fausse, mais n'a-telle pas subrepticement fait déraper l'enquête? À la question de savoir où commence et où s'arrête le rapport dit critique de Derrida à Heidegger, il semble que l'on ait très vite, trop vite, substitué la question de la justesse de la lecture derridienne de Heidegger: une question d'héritage légitime ou illégitime. Ce débat s'est polarisé en trois positions que je caricature un peu au bénéfice de l'illustration:

— Pour Derrida, contre Heidegger ${ }^{4}$.

— Contre Derrida, pour Heidegger ${ }^{5}$.

— Contre Derrida, contre Heidegger ${ }^{6}$.

Il serait grossier de prétendre que ce débat est sans intérêt. Mais la prise de parti n'est-elle pas prématurée? Comment évaluer si Derrida dilapide ou non l'héritage heideggérien avant même de nous être entendus sur la question de savoir en quoi consiste précisément le legs de Heidegger à sa pensée ? Car en tout premier lieu, ce dont il s'agit n'est pas de trancher à savoir si la lecture de Derrida est fidèle ou infidèle à la pensée de Heidegger, mais de reconstituer la scène d'un héritage.

Levée du rideau sur la scène. Dans celle-ci les rôles seraient, dit-on, bien établis: Heidegger, le penseur du rassemblement d'un côté; Derrida, le penseur de la dissémination de l'autre. Chacun chez soi, chacun occupant son côté de la scène. C'est l'évidence d'une telle ligne de partage, clairement tracée et délimitée, qui sera interrogée ici, et d'abord pour éviter que l'évidence, pour jouer de l'anglais, devienne trop rapidement et sans doute trop facilement une pièce à conviction (evidence) contre Heidegger.

Non qu'il faille soustraire ce dernier à l'accusation (la scène d'un héritage est toujours aussi la scène d'un procès), mais l'on risque d'incriminer

3. Jacques Derrida, Geschlecht III. Sexe, race, nation, humanité, Paris, Seuil, 2018, p. 161.

4. Je m'avance à donner quelques exemples: Richard Rorty, "Derrida on Language, Being and Abnormal Philosophy", Journal of Philosophy, vol. 74, n 11, 1977, p. 673-681; Briankle G. Chang, "The Eclipse of Being: Heidegger and Derrida", International Philosophical Quarterly, $\mathrm{n}^{\circ} 25,1985$, p. 113-137; Eugenio Donato, «Ending/Closure: On Derrida's Edging of Heidegger ", Yale French Studies, n 67, 1984, p. 3-22.

5. David Wood, "Heidegger after Derrida ", Research in Phenomenology, $\mathrm{n}^{\circ} 17,1987$, p. 103-116; Françoise Dastur, "Réception et non-réception de Heidegger en France", Revue germanique internationale, $\mathrm{n}^{\circ} 13,2011$, p. 37-55.

6. Jürgen Habermas, Le discours philosophique de la modernité, Paris, Gallimard, 1988, p. 214; Luc Ferry et Alain Renaut, La pensée 68. Essai sur l'anti-humanisme contemporain, Paris, Gallimard, 1985, p. 46-49, p. 189-191 et p. 281-285. 
Heidegger pour les mauvais motifs et de rater la véritable cible de Derrida. C'est à la fois notre héritage de Heidegger et notre héritage de Derrida qui risqueraient de s'en trouver dilapidés.

Et pourtant, l'évidence pèse lourdement. Il serait facile de le vérifier, le schème du rassemblement est à l'œuvre chez Heidegger à trop d'endroits pour qu'on puisse en faire l'inventaire rapide. Je me contente de pointer vers quelques-uns de ces lieux: l'esprit comme ce qui a la caractéristique de rassembler ou de se rassembler, le pont comme ce qui rassemble les deux rives, la lecture comme ce qui recueille les fruits, le quadriparti qui rassemble le ciel et la terre, les mortels et les dieux ${ }^{7}$. Le même schème intervient lorsque Heidegger discute de la cruche, du lieu, de la main, de l'oreille, de la pensée et de la mémoire ${ }^{8}$. L'évidence est trop massive pour qu'on la récuse simplement.

Elle se trouve en outre aggravée par l'intérêt que Derrida porte à ce schème du rassemblement. Là encore, nul besoin d'inventaire exhaustif, mais on pourrait probablement argumenter que chaque fois que Derrida discute d'un texte de Heidegger, le fil conducteur de sa lecture est réglé sur ce schème. Pas d'inventaire donc, mais uniquement les textes les plus évidents. Or, il se trouve que les textes les plus évidents sont aussi ceux qui connaissent un regain d'intérêt aujourd'hui' à la suite de la publication longtemps attendue de Geschlecht III. Sa publication nous permet d'embrasser de façon synoptique le projet Geschlecht (I à IV) et d'en apprécier la cohérence d'ensemble.

La série Geschlecht se déploie - parmi d'autres lectures possibles - comme une vaste interrogation du phénomène "rassemblement» chez Heidegger. Je mets des guillemets à «rassemblement» ici pour mimer le geste que fait Derrida quant à "Geschlecht", le mot ou la chose

7. Respectivement Martin Heidegger, Holderlins Hymne «Der Ister» (été 1942), dans Gesamtausgabe, Bd. 53, et Schelling. Traité de 1809 sur l'essence de la liberté humaine, Paris, Gallimard, 1977, p. 221; "Bâtir habiter penser", Essais et conférences, Paris, Gallimard, 1976, p. 170-193; Qu'appelle-t-on penser?, Paris, Presses Universitaires de France et «Logos », Essais et conférences, p. 251; «La chose ", Essais et conférences, p. 194-223.

8. Voir en particulier, Heidegger, Essais et conférences, p. 194-211 et p. 249-278; Martin Heidegger, "La parole dans l'élément du poème", Acheminement vers la parole, Paris, Gallimard, 1976, p. 39-84; Heidegger, Qu'appelle-t-on penser?, p. 90 et 146.

9. Cette référence à l'aujourd'hui trahit la provenance occasionnelle de ma contribution. Son impulsion lui a été donnée par la publication récente (octobre 2018) de Geschlecht III: sexe, race, nation, humanité et par une invitation à lire ce texte lancée par Ginette Michaud et Georges Leroux à l'occasion de l'édition 2018 des journées annuelles Mémoires de Jacques Derrida. N'étant alors pas de taille à me mesurer frontalement à Geschlecht III, j'avais pris prétexte de sa publication pour offrir plutôt une lecture transversale du projet Geschlecht dans son ensemble. Plus largement, ma contribution s'inscrit dans un projet postdoctoral (FRQSC 2017-2019) dont l'effort vise à mesurer l'apport heideggérien à la pensée de Jacques Derrida à la lumière des inédits de ce dernier. 
"Geschlecht $t^{10}$ ", pour marquer symétriquement qu'il est difficile de savoir au premier abord de quoi il est question lorsqu'on dit «rassemblement». Faut-il l'assimiler à un schème interprétatif, à une figure du discours, à une métaphore fondamentale ${ }^{11}$, à une valeur privilégiée par Heidegger ou encore à une axiomatique? Cela est difficile à déterminer non seulement parce que cela oscille d'un texte de Derrida à l'autre, mais surtout parce que la même oscillation affecte les textes de Heidegger.

L'hypothèse qui aimantera ma lecture est que ces différents phénomènes de «rassemblement» n'ont pas tous la même valeur pour Derrida, qu'il y a en somme une figure du rassemblement à questionner et une autre figure, plus grave mais aussi plus rare, à accuser. L'objectif du présent article sera donc de caractériser ces deux niveaux plus en détail et de les lier à la scène de l'héritage. On verra la valeur de rassemblement apparaître tour à tour de l'un et de l'autre côté de ces lignes de partage, et pluraliser le legs devenir l'enjeu de la scène.

Une note avant d'aller plus loin. Plus qu'une hypothèse ou une démonstration, cet article propose un parcours de lecture. Bien qu'un tel parcours ne soit pas aléatoire et qu'il laisse éprouver une certaine nécessité, il serait absurde de prétendre qu'il soit le seul possible. Voici donc les paramètres — je ne dis pas le protocole - qui auront, en rétrospective, réglé son pas et sa démarche, et qui permettront peut-être au lecteur de retracer en pointillé le chemin parcouru. Au départ, une décision: celle de tenter de lire le rapport entre Heidegger et Derrida à partir des archives de ce dernier, et en posant l'hypothèse que les cours et séminaires pourraient bien être le lieu où ce rapport est le plus lisible. Au sein de cet ensemble, une période a été circonscrite qui va de 1971 à 1989 (soit des séminaires «La famille de Hegel (1971-1972)» à «Politiques de l'amitié (1988-1989)»). Le choix de cibler cette période se règle sur une affirmation où Derrida reconnaît sa dette, sinon à l'égard de Heidegger, du moins à l'égard d'une question heideggérienne, celle du don (es gibt, die Gabe, das Geben), au sujet de laquelle il écrit qu'elle "oriente expressément tous les textes que j'ai publiés depuis 1972 environ $^{12} »$.

Au sein de ce corpus, une place privilégiée a été accordée à la série Geschlecht, dont tous les morceaux, à l'exception du premier, auront d'abord été des cours ( «Le Fantôme de l'autre: Nationalité et nationalisme philosophiques» pour Geschlecht II et III, «Politiques de l'amitié» pour Geschlecht $I V)$. Ce privilège est circonstanciel ${ }^{13}$ et ne s'éclairera qu'à convo-

10. Jacques Derrida, Psyché. Inventions de l'autre, Paris, Galilée, 1987, p. 395. Voir aussi Derrida, Geschlecht III, p. 39, note 2.

11. Sur le modèle des «paroles fondamentales » qui composent le livre de Marlène Zarader, Heidegger et les paroles de l'origine, Paris, Vrin, 1986, p. 18-19.

12. Derrida "Dénégations", Psyché, p. 587. Voir aussi Jacques Derrida, Donner le temps I. La fausse monnaie, Paris, Galilée, 1991, p. 10.

13. Voir ci-dessus, note 9. 
quer beaucoup d'autres cours, publiés ou non ${ }^{14}$. Car ce qui m'a paru le plus remarquable dans tous ces cours, c'est que Derrida s'y débat littéralement avec le legs heideggérien. C'est ce débat et ce «se débattre ( "nous ne serons jamais débarrassés du corps de Heidegger ", dixit Derrida) que j'ai voulu mettre en scène ici, exemplairement quant au motif du rassemblement, si lancinant dans l'Auseinandersetzung entre Derrida et Heidegger ${ }^{15}$.

\section{Le rassemblement: une insistance à questionner, une nécessité à méditer}

Repartons du début. Le rassemblement se partage. Il peut être à questionner ou à accuser. Le rassemblement à questionner est présent jusque dans le titre de la série Geschlecht, puisque dans sa plus grande formalité "Geschlecht » signifie le "nous» d'une communauté, d'un être-rassemblé (famille, genre, espèce, etc.), mais aussi, disons-le, d'une communauté dans ou à travers la dispersion, sous le mode de la filiation et de l'héritage ${ }^{16}$.

À partir de "La main de Heidegger (Geschlecht II) ", l'insistance de ce motif du rassemblement au sein des textes heideggériens devient un des points focaux de la série. Citons en cascade Geschlecht II, III et IV pour mettre en évidence la récurrence, mais aussi une certaine radicalisation du propos de Derrida:

Geschlecht II: "C'est toujours le rassemblement que privilégie Heidegger ${ }^{17}$."

Geschlecht III: "Nous touchons ici à ce que d'autres appelleraient "l'axiomatique" de la "lecture" de Heidegger: il y a ou doit y avoir du rassemblement ${ }^{18}$.»

Geschlecht $I V:$ «[...] cette force de rassemblement qui rassemble en vérité toute l'œuvre de Heidegger ${ }^{19}$.»

On pourrait sans mal ajouter à cette liste De l'esprit..., qui s'inscrit dans le droit fil de la série Geschlecht ${ }^{20}$. Derrida y adresse quatre questions à Heidegger (formalisées par ailleurs dans «On Reading Heidegger ${ }^{21}$ ») sur la «question", l'animalité, l'essence de la technique et l'épochalité. S'étant demandé ce qui pouvait bien nouer ensemble ces quatre fils, Derrida y

14. Lorsque référence est faite à des cours et séminaires inédits, j’indique la localisation du matériel dans la collection des Special Collections and Archives de l'University of California, Irvine, où je les ai consultés. Je remercie le personnel de cette institution pour son accueil chaleureux.

15. Pour une mise en scène différente de ce même débat, voir l'excellent article de Jussi

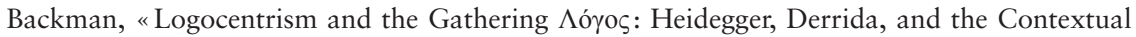
Centers of Meaning ", Research in Phenomenology, n 42, 2012, p. 67-91.

16. Voir Derrida, "Geschlecht. Différence sexuelle, différence ontologique », Psyché, p. 395 et p. 400 ; Derrida, «La main de Heidegger (Geschlecht II) », Psyché, p. 417.

17. Derrida, "La main de Heidegger (Geschlecht II) », Psyché, p. 439.

18. Derrida, Geschlecht III, p. 47.

19. Jacques Derrida, Politiques de l'amitié, Paris, Galilée, 1994, p. 395.

20. Voir Krell, Phantoms of the Other, p. 17.

21. Jacques Derrida, "On Reading Heidegger: An Outline of Remarks to the Essex Colloquium ", Research in Phenomenology, n 17, 1987, p. 171-185. 
répond magistralement par l'esprit dont le motif organise alors toute sa lecture de Heidegger ${ }^{22}$. Or la caractéristique assignée à l'esprit par Heidegger est précisément de rassembler ou d'être l' "unité originellement unifiante ${ }^{23}$ ».

Cette insistance sur le motif du rassemblement a de quoi surprendre et peut sembler au premier abord arbitraire. Dans le séminaire «La chose II (Heidegger/Blanchot) ", Derrida met en parallèle deux lectures du pont, l'une chez Heidegger, où il rassemble les deux rives, l'autre chez Blanchot, où il disloque et rend inaccessible l'autre rive ${ }^{24}$. Compte tenu des affinités bien connues de Derrida avec Blanchot, on aurait pu s'attendre à ce qu'il se serve de ce contraste pour jouer Blanchot contre Heidegger et contre le caractère arbitraire de son interprétation rassemblante du pont. Or il n'en est rien, et la suite du tapuscrit ne laisse transpirer aucune prise de parti de la part de Derrida. Cela indique déjà de manière préliminaire que le schème interprétatif du rassemblement ne doit pas être considéré en lui-même comme fautif. Si un tel schème peut bien devenir, à la longue, monotone et sujet de moqueries, si son insistance peut à bon droit devenir l'objet d'un questionnement en bonne et due forme, ce n'est pas nécessairement un geste interprétatif fautif.

Et pourquoi pas ? Parce que Derrida reconnaît une certaine nécessité à ce geste interprétatif là où Heidegger médite la question du il y a (es gibt $)^{25}$, c'est-à-dire là où il est question du don, qui a aussi été pendant longtemps au centre des intérêts de Derrida ${ }^{26}$. Puisqu'il ne s'agit pas ici d'élucider la pensée heideggérienne de l'Ereignis, je dirai seulement que Heidegger pose l'hypothèse que, pour qu'il y ait en général, pour que quelque chose ait lieu ou arrive au sens large, il faut du rassemblement. Le rassemblement et l'avoir-lieu seraient en quelque sorte indissociable, cela figurerait une loi inattaquable de la phénoménalité: pas de don sans rassemblement.

22. Jacques Derrida, De l'esprit. Heidegger et la question, Paris, Galilée, 1987, p. 23-30. Pour une approche plurielle et minutieuse du projet de Derrida dans ce livre, voir le collectif dirigé par David Wood, Of Derrida, Heidegger, and Spirit, Evanston (IL), Northwestern University Press, 1993.

23. Heidegger, Schelling..., p. 221. Voir Derrida, De l'esprit, p. 122-123.

24. Collection Jacques Derrida. MS-C001. Boîte 13, dossier 12, feuillet 5 (séance 3). Special Collections and Archives, The UC Irvine Libraries, Irvine, California. Archives consultées en juillet-août 2018. Derrida se réfère à Heidegger, "Bâtir, habiter, penser ", Essais et conférences, p. 180-181 et vraisemblablement à Maurice Blanchot, "L'interruption ", L'entretien infini, Paris, Gallimard, 1969, p. 110. Merci à Cosmin Popovici-Toma pour le repérage de cette référence. Dans «Pas» (Parages, Paris, Galilée, 1986, p. 19-116), Derrida propose une lecture de la rive inatteignable (impossible abordage, arrivée sans rivages, etc.) dans les récits de Blanchot (en particulier Le pas au-delà, Thomas l'Obscur, Celui qui ne m'accompagnait pas), sans y mobiliser explicitement la figure du pont, mais où l'enjeu est le même (voir Derrida, Parages, p. 65.)

25. Voir en particulier Martin Heidegger, "Temps et Être ", dans Questions III et IV, Paris, Gallimard, 1990, p. 189-225.

26. Ibid., p. 4. 
Cela mérite qu'on s'y attache un peu. Réfléchissant au sens du titre Etre et Temps, Heidegger indique dans un de ses cours: " "Être", ça va; "temps", ça va aussi; mais "être et temps" ? Le "et" qui les comprime ensemble est l'index

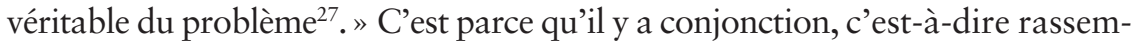
blement - Derrida dira pour sa part articulation ${ }^{28}$ - qu'il y a être (es gibt Sein), qu'il y a temps (es gibt Zeit). À la condition de prendre le rassemblement pour ce qu'il est, c'est-à-dire une métaphore, on peut voir en lui une manière de répondre à la question de l'origine (création, fondement, naissance) pensée comme fondement abyssal, une manière qui vise à y répondre hors des régimes théologique, mythologique, métaphysique et anthropologique. Plus précisément, la métaphore du rassemblement permet à Heidegger de représenter l'opération par laquelle "il y a " quelque chose plutôt que rien - l'opération par laquelle «il y a » de l'être, de la pensée, de l'histoire, du Dasein, et peut-être même de l'homme.

Comme support analogique, le rassemblement décrit, illustre ou évoque alors ce-qui-se-passe-lorsqu'il- $y$ - $a$ (ce qui doit se passer pour qu'il y ait); non pas le quoi?, le pourquoi? ou le qui? du «il y a », mais son comment? Le rassemblement serait donc le "moteur» de la phénoménalité, c'est-à-dire ce sans quoi il y aurait «rien » plutôt que «quelque chose».

\section{Don, rassemblement, économie: la scène partagée}

On pourrait croire que Derrida, le penseur de la dissémination, s'opposerait de façon frontale à cette hypothèse heideggérienne. Rien ne saurait être plus éloigné de la réalité. Derrida est au contraire absolument fasciné par cette hypothèse et par la régularité troublante avec laquelle elle se vérifie en pratique.

On dira pourtant que le motif du rassemblement est absent des textes de Derrida qui ne portent pas sur Heidegger. C'est vrai si l'on considère que son absence thématique signifie aussi son absence pure et simple. Or, si l'on s'entend sur le fait que l'opération décisive du rassemblement est celle d'une convergence ou d'un "ça revient au même ", il faut alors dire que ce schème

27. Martin Heidegger, De l'essence de la liberté humaine. Introduction à la philosophie, Paris, Gallimard, 1987, p. 115-116.

28. C'est d'ailleurs, notons-le au passage, ce fil conducteur de la conjonction/articulation comme ce qui fait advenir et comme avoir-lieu qui organise tout le propos de Derrida dans le cours Heidegger, la question de l'Être et l'Histoire: cours de l'ENS-Ulm 1964-1965 (Paris, Galilée, 2013, p. 43-44): "Il nous faudra parler de l'histoire, c'est-à-dire du “et”, c'est-à-dire du lieu de la communication et du passage entre l'être et l'histoire; ce passage, ce "et" étant le lieu même de notre problème, ce "et" dont il n'est pas encore décidé si nous l'écrirons "et" ou “est". " À peine quelques années plus tard, alors qu'il cherche à caractériser l'opération de la différance, Derrida écrit dans De la grammatologie (Paris, Minuit, 1967) qu'elle — la différance - «permet à la différence entre l'espace et le temps de s'articuler, d'apparaître comme telle dans l'unité d'une expérience [...] C'est de la possibilité première de cette articulation qu'il faut partir. La différence est l'articulation" (p. 96, je souligne). 
est massivement présent chez Derrida tout au long de son œuvre sous le thème de l'économie ${ }^{29}$. La différance, par exemple, est par excellence le «concept" même de l'économie à l'œuvre, à la fois sa ressource et son frein ${ }^{30}$. Pour une part non négligeable d'eux-mêmes, rassemblement et différance visent la même opération.

Ce schéma économique perfuse non seulement un très grand nombre de textes de Derrida (à commencer par «Violence et métaphysique » et «De l'économie restreinte à l'économie générale $\left.{ }^{31} »\right)$, mais il organise aussi presque systématiquement tous ses cours et séminaires des années 1970 et 1980, au moins jusqu'au moment de son passage à l'École des hautes études en sciences sociales. Sous des thèmes aussi disparates que ceux de la dépense, du propre, de la famille, de la sépulture, de la femme, du don ou du deuil, Derrida paraît traquer d'un cours à l'autre la même structure circulaire, le même mouvement (possible-impossible) d'un retour au même. Je me limite à deux exemples tirés des cours.

\section{Le don}

À première vue le don, motif emblématique de la pensée derridienne que l'on peut d'ailleurs associer à la dissémination — don sans retour —, n'a pas sa place à titre d'exemple de la logique économique. Le phénomène du don se laisse en effet résumer par sa gratuité. Et pourtant, s'il y a bien quelque chose qui ressort de «Donner le temps ${ }^{32}$ » (1977-1978), c'est que, non seulement le don semble voué à réintégrer le circuit de l'échange (l'exemple de Mauss et du potlatch étant ici paradigmatique), mais pis encore il semble se détruire comme don dès qu'il est reconnu comme tel par le donateur ou le

29. Je renvoie à la littérature secondaire abondante qui a exploité cette affinité entre la déconstruction et l'économie: Irène Harvey, Derrida and the Economy of Differance, Bloomington, Indiana University Press, 1985; Joost de Bloois, «The Last Instance: Deconstruction as Economy ", dans Sjef Houppermans et al. (dir.), Enduring Resistance: Cultural Theory after Derrida, Amsterdam, New York, Rodopi, 2010, p. 121-138; Robert Bernasconi, "What Goes Around Comes Around ", dans Alan Schrift (dir.), The Logic of the Gift: Towards an Ethics of Generosity, London, Routledge, 1997, p. 256-273; Egidius Berns, "Pourquoi Derrida? ", Rue Descartes, vol. 82, n 3, 2014, p. 22-25; Nicholas Cotton, Économie de la perversité baudelairienne. Une lecture de Donner le temps de Jacques Derrida, Mémoire de maîtrise en littératures de langue française, Université de Montréal, 2013, p. 20-25 et p. 67-116; Stephen Pluhacek, Economy and Strategy: From Timaeus to Différance, Thèse de doctorat en philosophie, Purdue University, 1999, p. 119 sq.

30. Jacques Derrida, "La différance", dans Marges — de la philosophie, Paris, Minuit, 1972 , p. 8 , p. 14 , p. $18-19$ et surtout p. 20-21.

31. Tous deux repris dans Jacques Derrida, L'écriture et la différence (Paris, Galilée, 1967).

32. Puisque ce cours a été repris et publié sous un titre identique (Donner le temps), je cite ce dernier. Pour un résumé synthétique de la problématique du don, voir Jacques Derrida, «Une certaine possibilité impossible de dire l'événement », dans Alexis Nouss, Gad Soussana, et Jacques Derrida, Dire l'événement, est-ce possible? Séminaire de Montréal pour Jacques Derrida, Montréal, Paris, L'Harmattan, 2001, p. 92-93. 
donataire. "C'est en ce sens peut-être que le don est l'impossible ${ }^{33}$ » même, dit Derrida, car en tant qu'acte il ne peut manquer d'instituer sa dette (matérielle ou symbolique) et d'initier une demande de restitution. Il y a donc contradiction entre la condition de possibilité du don (la gratuité) et le phénomène «don", qui s'annule ou s'investit presque invariablement dans l'échange économique.

\section{La sépulture}

La mort semble elle aussi devoir être considérée comme la figure anéconomique par excellence, car elle signifie la perte pure et simple, sans retour possible. Il est vrai que, dans la dialectique du maître et de l'esclave hégélienne par exemple, l'exposition à la mort produit une plus-value qui compense le risque encouru et le réinscrit dans un cercle économique: «Il faut risquer de perdre pour gagner », telle serait la maxime de la négation déterminée ${ }^{34}$. Mais, fatalement, la mort est inévitable et il faut en tenir compte. Dans le cours intitulé «La famille de Hegel $^{35}$ » (1971-1972), Derrida veut montrer comment chez Hegel, même la mort, figure absolue de la perte et de la dépense sans réserve, peut encore être réappropriée par l'intermédiaire du rite de la sépulture ${ }^{36}$. Celle-ci relève (aufgehoben) littéralement la perte en empêchant le défunt de passer à l'état de chose anonyme. Lorsqu'Antigone proteste contre la décision de Créon de laisser son frère sans sépulture, c'est cet abandon du mort aux forces de la matière qui est accusé comme manquement à la loi naturelle. Le mort ne doit pas devenir un simple cadavre. La sépulture vient signifier à la fois que la vie de l'esprit résiste à la mort et que l'appartenance du mort à la communauté humaine n'est pas brisée. Le défunt demeure.

Ces deux exemples témoignent d'un patron de lecture omniprésent durant la période où Derrida enseigne à l'ENS. À chaque fois, il s'agit de prendre comme fil conducteur une figure de l'économie pour mettre en évidence à la fois sa nécessité et ses limites non accidentelles. Derrida s'explique justement au sujet de son «obsession» économique dans le cours intitulé

33. Derrida, Donner le temps, p. 19.

34. Je rappelle le célèbre passage de la préface de la Phénoménologie de l'esprit: «La mort, si nous voulons nommer ainsi cette ineffectivité, est ce qu'il y a de plus redoutable, et tenir fermement ce qui est mort [est] ce qui exige la force la plus grande. [...] ce n'est pas la vie qui s'épouvante devant la mort et se garde pure de la dévastation, mais celle qui la supporte et conserve dans elle qui est la vie de l'esprit. Il ne gagne sa vérité qu'en tant qu'il se trouve luimême dans le déchirement absolu. [...] Ce séjourner est la forme magique qui le convertit dans l'être» (trad. fr. G. Jarczyk et P. J. Labarrière, Paris, Gallimard, 1993, tome 1, p. 46-47).

35. Repris dans Jacques Derrida, Glas, Paris, Galilée, 1974. Sur la sépulture, voir p. $161-165$.

36. Dans un cours intitulé Dieu, la mort et le temps (Paris, Grasset et Fasquelle, 1993, p. 97-103), Lévinas commente le même passage de Hegel sur la sépulture. 
«La langue et le discours de la méthode» (1981-1982). Il ouvre la 6 e séance par cette justification:

Ma première phrase aujourd'hui a été pour dire qu'une fois de plus, une fois de plus, mon interprétation se confiait à une certaine pensée de l'économie. Une fois de plus - parce que vous savez, certains d'entre vous savent que c'est là un geste assez insistant de ma part, au point de pouvoir paraitre monotone, à moins qu'on ne le crédite du fait que l'économie justement insiste elle-même partout, qu'elle est elle-même assez une loi (oikonomia) pour appeler une loi économique d'interprétation. [...] Mais ce n'est pas tout. Car d'une part, analyser l'économique, c'est-à-dire, comme nous l'avons vérifié une fois de plus, une sorte de circularité, de cercle faisant retour à son point de départ, c'est aussi mettre en évidence l'anéconomique, c'est-à-dire ce qui ou bien empêche régulièrement le cercle de se fermer ou bien le relance sans fin ${ }^{37}$.

«[L]'économie [...] insiste elle-même partout». Autre façon de dire que le rassemblement n'est pas quelque chose contre quoi il faudrait prendre parti mais quelque chose avec quoi il faut composer. Pas le choix ici, il faut gérer l'économie.

Je voudrais mettre en évidence un moment remarquable où Derrida est rappelé à cette loi économique. Cela se passe dans Théorie et pratique, au début de la septième séance. Derrida annonce qu'il prend son congé vis-à-vis de sa pratique pédagogique classique, celle où il lit et performe un texte préalablement écrit et travaillé. Il le fait, dit-il, pour faire droit à l'injonction qui fait tout l'enjeu du séminaire: ne plus calquer la pratique sur une théorie qui la surveille. Derrida risque alors l'improvisation et plie sa pratique enseignante à la règle sans règle de l'anéconomique ${ }^{38}$.

Comment tout cela va-t-il se passer? Eh bien, mal, pourrait-on dire, ou à tout le moins s'agit-il d'une expérience en demi-teinte. Nous sommes pratiquement au même endroit du cours, Derrida n'a guère avancé que de quelques lignes et s'interrompt alors pour déclarer:

J'en étais là de la préparation de cette séance quand je me suis aperçu, sans grande surprise d'ailleurs - que sous prétexte de transformer davantage, de frayer davantage, de multiplier les risques et les chances, ma nouvelle pratique qui consistait à improviser à partir de notes était non seulement une fiction mais un dispositif invitant à la répétition [...] et en somme revenait à une perte de travail, de force de frayage et de travail. [...] Se libérer - du moins dans cette situation - du travail d'écriture me paraissant être une mystification et une facilité, allant à l'encontre même de ce qu'on recherche, je reviens, au moins provisoirement, à mon ancienne pratique, au moins par moments ${ }^{39}$.

37. Collection Jacques Derrida. MS-C001. Boite 16, dossier 11, feuillet 1 (séance 6). Special Collections and Archives, The UC Irvine Libraries, Irvine, California. Archives consultées en juillet-août 2018.

38. Jacques Derrida, Théorie et pratique. Cours de l'ENS-Ulm 1975-1976 [sic], Paris, Galilée, 2017, p. 140.

39. Ibid., p. 140-141. 
L'économie insiste. Tout bien considéré, l'improvisation est improductive, elle est une figure de la perte de temps et d'efficience. Il faut donc l'économie, une certaine organisation réglée, pour éviter que la pratique enseignante soit stérile - stérilité qui est toujours un risque de la dissémination. Derrida annonce donc qu'il fera sa part à l'économie. Il s'agit ici littéralement d'une économie de l'économie, d'un certain équilibre entre la rigidité économique qui calcule et planifie jusque dans les moindres détails pour maximiser ses profits et l'improvisation anéconomique qui risque la dissémination en pure perte. Il y aurait donc, on le voit bien, quelque naïveté à ranger Derrida purement et simplement du côté de la dissémination, comme s'il en était le chantre et comme si cela avait un sens d'être «du côté » de la dissémination.

\section{De la question à l'accusation : la division de l'héritage}

Où situer alors la ligne de partage et éviter la confusion où nous ne serions plus en mesure de distinguer la mise en question de la mise en accusation? La série Geschlecht - et tout particulièrement Geschlecht III - nous permet de mieux répondre à cette question. Je lirai la série comme une scène d'héritage et une scène à propos de l'héritage, dont l'enjeu porte au fond sur ce qu' « hériter » veut dire, sur ce que ça doit vouloir dire pour l'un des protagonistes et sur ce que ça peut vouloir dire pour l'autre.

À quoi avons-nous affaire avec Geschlecht III? À Derrida lisant Heidegger lisant Trakl. Scène d'héritage abyssale, s'il en est. "Que faut-il hériter de Trakl ? ", demande Heidegger; "que faut-il hériter de Heidegger lisant Trakl? », demande Derrida. Et vous voilà, me lisant ici lire Derrida lire Heidegger lire Trakl en vous demandant ce qu'il faut en hériter. Comme tout abîme, celui-ci s'accompagne d'un vertige.

Devant un tel abîme, une seule solution possible, celle de sauter. Cela, nul doute, c'est de Heidegger que nous l'héritons. La valeur herméneutique que ce dernier attribue au saut ${ }^{40}$, et donc à un certain pôle anéconomique dans sa démarche de lecture, est bien connue. Ce recours au saut n'est pas étranger à la difficulté singulière du texte de Heidegger sur Trakl, qui est si déroutant - et même pour certains carrément inadmissible si on l'évalue à partir de normes philologiques classiques ${ }^{41}$. Or ce n'est pas un hasard si Derrida, dans ce qu'il appelle une «longue parenthèse ${ }^{42}$ » au milieu de la neu-

40. Derrida, Geschlecht III, p. 46. Voir Martin Heidegger, "Identité et différence», Questions I et II, Paris, Gallimard, 1990, p. 266 et Le principe de raison, Paris, Gallimard, 1962, p. 146-147.

41. Pour ne pas surcharger inutilement le lecteur, je renvoie à l'article de Rémy Colombat qui résume très bien les données fondamentales du débat (et prend parti): «Heidegger, lecteur de Trakl", Études germaniques, vol. 271, n 3, 2013, p. 395-420. Pour une position sensiblement différente, voir l'étude classique de Beda Allemann, Hölderlin et Heidegger, Paris, Presses Universitaires de France, 1959, p. 12-16 et 243-282.

42. Derrida, Geschlecht III, p. 86-87. 
vième séance de Geschlecht III, en vient à remarquer que Heidegger lisant Trakl nous donne à lire sa propre situation énonciative. De la même manière que le dément, dans le poème de Trakl, est «en chemin vers ailleurs» et non pas extravagant ou errant sans direction, l'interprétation heideggérienne procède par sauts et par bonds sans exclure un être-en-chemin. Derrida évalue très positivement cette démarche qui se situe, qui dit d'où elle vient, où elle va et comment elle y va, bref qui ne dissimule pas l'intérêt qui la fonde et qui n'a pas peur d'amener le texte ailleurs - quitte à passer pour dément.

Que l'interprétation de Heidegger tende à rassembler la poésie de Trakl en un tout cohérent, et que le point focal de cette interprétation soit un certain schème du rassemblement, cela se laisse à bon droit questionner. Geschlecht III, De l'esprit, sont les archives les plus parlantes d'un tel dialogue virtuel entre Derrida et Heidegger. Mais il ne faut pas lire trop vite l'accusation dans la question. "De mon inquiétude, dira Derrida dans "Désistance", je ne ferai pas une critique, car je ne crois pas évitable ce geste de rassemblement. Il est toujours productif et philosophiquement nécessaire ${ }^{43}$.»

Ce geste interprétatif "positif» s'accompagne cependant parfois chez Heidegger, et assez fréquemment pour que Derrida le dise «typique ${ }^{44}$ », d'une procédure de légitimation par laquelle il tente de faire valoir la nécessité de son interprétation. Ce geste se conçoit comme prétention à l'héritage exclusif, et en ce sens il va radicalement à l'encontre de la démarche autosituante dont Derrida faisait plus tôt l'apologie, démarche qui concevait l'héritage comme un legs partagé.

Marquons une pause ici pour interroger cette «typicité» du geste heideggérien. Cette caractérisation est sans doute le lieu de Geschlecht III où l'argumentation de Derrida est la plus fragile, en tout cas la plus exposée à la contestation. À partir de quel moment un geste devient-il typique? Quels sont les critères, quantitatifs et/ou qualitatifs, qui permettent de conclure à une telle typicité ? Cela est d'autant plus énigmatique que Derrida avait luimême mis en garde contre la tentation d'assimiler le texte heideggérien à un bloc homogène, cohérent et unidirectionnel dans son intention. Derrida écrit à cet effet dans "Geschlecht II " qu'il "ne "critique" jamais Heidegger sans rappeler qu'on peut le faire depuis d'autres lieux de son texte [celui de Heidegger]. Celui-ci ne saurait être homogène et il est écrit à deux mains, au moins ${ }^{45}$ ». À l'époque de "Geschlecht II », Derrida considère encore que le texte de Heidegger est travaillé par des tensions contradictoires, chaque main tirant l'étoffe de son côté au risque de la déchirer. À partir de Geschlecht

43. Derrida, «Désistance », Psyché, p. 616. Je souligne.

44. Derrida, Geschlecht III, p. 38-42.

45. Derrida, «La main de Heidegger (Geschlecht II) », Psyché, p. 447. 
III, l'une des deux mains de Heidegger semble l'emporter ${ }^{46}$ et l'un des deux gestes devenir assez régulier ou récurrent pour être nommé «typique », c'està-dire emblématique, caractéristique, etc., de la signature "Heidegger». Il serait certes injuste de crier à l'incohérence de la part de Derrida, mais ne fait-il pas, sur le plan de l'argumentation, exactement ce qu'il reproche à Heidegger lorsqu'il en vient à déclarer dans "Geschlecht IV" que le motif du rassemblement «rassemble en vérité toute l'œuvre de Heidegger ${ }^{47}$ »? À quel moment un geste devient-il assez typique pour rassembler «en vérité » toute une œuvre? Une pensée de la typicité ou de l'itérabilité ne devraitelle pas exclure par principe ce genre de totalisation? Un cheminement typé n'autorise-t-il pas par principe certaines altérations de la démarche? N'est-ce pas même ce dérèglement contradictoire que Derrida nous a appris à repérer dans les textes sous le nom de déconstruction? Or, de la typique (régularité, etc.) à la systématique ( «toute l'œuvre»), il y a un pas que dans «Geschlecht IV » Derrida n'hésite plus à franchir et qui franchement s'explique mal. Fin de la pause.

Quoi qu'il en soit, dans Geschlecht III Derrida veut mettre en évidence la faiblesse inhabituelle de l'argumentation heideggérienne qui cherche à récuser l'interprétation chrétienne de Trakl ${ }^{48}$. Le dialogue remarquable qu'il imagine dans De l'esprit... entre Heidegger et des théologiens chrétiens ${ }^{49}$ vise justement à montrer que leurs interprétations respectives sont loin d'être mutuellement exclusives, au moment même où Heidegger voudrait faire admettre que le Gedicht de Trakl ne promet aucun héritage chrétien, et cela parce que le Gedicht en général ne peut ou ne doit avoir qu'un seul lieu propre, une seule destination, car il n'a au fond qu'une seule origine. "Tout grand poète, dit Heidegger à propos de Trakl, n'est poète qu'à partir de la dictée d'un unique Gedicht ${ }^{50}$."

Il n'est pas inopportun de noter qu'un schéma tout à fait similaire est à l'œuvre dans le séminaire de 1975-1976 intitulé «La vie la mort ». Lors de la huitième séance, Derrida commente la réaction défensive et même crispée de Heidegger quant à l'interprétation biologique ou biologisante de Nietzsche ${ }^{51}$. La question est importante, puisqu'on sait à quelles associa-

46. «Si le lieu est régulièrement, typiquement défini par le rassemblement (Versammlung), toute notre approche du geste heideggérien devra questionner ce privilège du rassemblement et tout ce qu'il induit. » Derrida, Geschlecht III, p. 42.

47. Derrida, Politiques de l'amitié, p. 395. Je souligne.

48. Heidegger, Acheminement..., p. 77-78. Derrida, Geschlecht III, p. 108-117.

49. Derrida, De l'esprit, p. 178-184.

50. Heidegger, Acheminement..., p. 41-42.

51. Collection Jacques Derrida. MS-C001. Boîte 12, dossier 12 (séance 8). Special Collections and Archives, The UC Irvine Libraries, Irvine, California. Archives consultées en juillet-août 2018. Voir Jacques Derrida, La vie la mort. Séminaire 1975-1976, Paris, Seuil, p. 201-224. Derrida commente alors le Nietzsche I de Heidegger (Paris, Gallimard, 1971, p. 266 sq., p. $402-410$ et p. 458 sq.) 
tions douteuses cette interprétation a pu donner lieu ${ }^{52}$. Mais en tentant de sauver le legs nietzschéen, Heidegger met en œuvre un geste ambigu au moins dans ses conséquences: le sauvetage auquel il s'adonne risque de perdre peut-être plus sûrement encore le legs de Nietzsche. La ligne d'argumentation heideggérienne consiste, comme vis-à-vis de l'interprétation chrétienne de Trakl, à faire valoir la destination unique et unitaire de la pensée de Nietzsche. "Chaque penseur, écrit Heidegger dans Qu'appelle-t-on penser?, pense seulement une unique pensée ${ }^{53}{ }^{\prime}$. Cette requête de l'unique et de l'unité, donc du rassemblé, Derrida y voit le critère ultime et «typique » de l'interprétation heideggérienne. On pourrait aussi y voir le critère par lequel juger et disqualifier les héritiers concurrents.

Faisons un pas de côté. Il serait possible de relire rétrospectivement le motif de la Destruktion sous l'angle de l'héritage comme un geste visant à pallier le défaut d'héritage de la tradition. Il n'y a pas eu d'héritage, dirait Heidegger, voulant dire par là que la tradition a mal hérité, a mal fait son travail en tant qu'héritière de la pensée - en particulier de la pensée grecque - et d'abord parce qu'elle a méconnu ce qu'hériter veut dire.

Par analogie avec le bon et le mauvais coup (Schlag) qui frappe le Geschlecht ${ }^{54}$, on pourrait distinguer chez Heidegger un bon et un mauvais coup de l'héritage; l'un resterait à venir et à assumer, dans la forme peut-être du Gespräch (entretien, dialogue) entre le poète et le penseur ${ }^{55}$, l'autre traduirait le rapport inauthentique du Dasein à son être-historial ${ }^{56}$.

Le premier, ce que Heidegger appelle dans un texte sur Héraclite un «dialogue pensant ${ }^{57}$ ", engage le penseur à répondre et à correspondre avec la parole du poète ou du philosophe ${ }^{58}$, ce qui n'est pas sans rappeler le thème

52. Les thèmes biologistes des écrits de Nietzsche ont participé à la récupération national-socialiste de sa pensée. Sur cette question voir Walter Kaufman, Nietzsche Philosopher, psychologist, antichrist, Princeton, Princeton University Press, 1968. Voir aussi Steven E. Aschheim, "Nietzsche in the Third Reich", The Nietzsche Legacy in Germany: 1890-1990, Berkeley et London, University of California Press, 1992, p. 232-271. Heidegger écrit avoir donné ses cours sur Nietzsche (1936-1940) en réponse à l'appropriation nazie de Nietzsche (Martin Heidegger, «Lettre au Rectorat académique de l'Université Albert-Ludwig, 1945 », dans Michel Haar (dir.), Cahier de l'Herne. Heidegger, Paris, Éditions de l'Herne, 1983, p. 102).

53. Heidegger, Qu'appelle-t-on penser?, p. 47.

54. Derrida, Geschlecht III, p. 72-77 et p. 147-148. Voir aussi Derrida, Psyché, p. 402 et surtout p. 413.

55. Martin Heidegger, Approche de Hölderlin, nouvelle édition augmentée, trad. fr. H. Corbin, M. Deguy, F. Fédier et J. Launay, Paris, Gallimard, 1973, p. 49.

56. Heidegger décrit le double versant (authentique/inauthentique) de l'historialité du Dasein aux \$74-75 d'être et Temps. Il y conçoit l'historialité authentique comme délivrance d'un héritage à partir d'un "provenir originaire". La tâche corrélative de celui ou celle qui questionne en direction du passé se trouve décrite, bien sûr, au $\$ 6$ d'Être et Temps, soit celle d'une destruktion de l'histoire de l'ontologie.

57. Heidegger, "Alèthéia (Héraclite, fragment 16) ", Essais et conférences, p. 316.

58. Voir Heidegger, "Qu'est-ce que la philosophie? ", Questions I et II, p. 333. 
derridien de la contresignature. Mais encore une fois, la destination du dialogue y semble surdéterminée par la valeur de l'unité, du rassemblement:

L'être de l'homme a son fondement dans le langage; mais celui-ci ne prend une réalité-historiale authentique que dans le dialogue. [...] Mais que signifie donc un «dialogue»? Évidemment, le fait de parler les uns avec les autres sur quelque chose. Seulement Hölderlin dit: «Depuis que nous sommes un dialogue et que nous pouvons ouir les uns des autres ». [...] Nous sommes un dialogue, cela signifie en même temps toujours: nous sommes un dialogue. L'unité d'un dialogue consiste en ce que chaque fois, dans la parole essentielle, soit révélé l'Un et Même sur lequel nous nous unissons, en raison duquel nous sommes Un et ainsi authentiquement sommes nous-mêmes ${ }^{59}$.

Ce qui distinguerait alors les deux formes de l'héritage ou de l'hériter, c'est le geste du retour au propre: retour à la chose (Sache) propre d'une pensée (celle de Nietzsche, par exemple), au lieu propre d'un Gedicht (celui de Trakl ou de Hölderlin); ou encore, retour au plus propre que le propre, ce que Heidegger appelle l'impensé ou l'imprononcé (Ungesprochene). L'impensé nomme ici ce qui aurait $p u$, ce qui aurait $d \hat{u}$, être pensé mais ne l'a pas été ${ }^{60}$; le legs ne s'est pas constitué, et la tradition est demeurée en défaut d'héritage.

La disqualification des héritiers n'est pas un accident de l'argumentation heideggérienne. Il faut y voir une conséquence de la conception du legs comme quelque chose d'indivisible, et d'indivisible parce qu'il a une provenance unique et unitaire. Ce qui se laisse spécifiquement accuser chez Heidegger c'est finalement, dira Derrida dans «La vie la mort », "ce présupposé $[\ldots]$ selon lequel il doit y avoir une interprétation une, rassemblée, autour d'une pensée unifiant un texte unique ${ }^{61}$ ». Ce présupposé quant à la forme rassemblante de l'interprétation, quant à la forme, aussi, rassemblée de la pensée ou du Gedicht (un auteur, une intention pleinement maîtrisée, une œuvre achevée qui reflète ce projet, etc.), est le seul qui mérite pour Derrida l'accusation de "métaphysique ». Il s'agit d'un présupposé quant à la nature du legs (comme s'il était un, et comme s'il n'y en avait qu'un), tout autant - et symétriquement - qu'un présupposé quant à la nature de l'héritage, conçu comme geste de rassemblement en retour (ré-assemblement) à partir d'une possibilité originaire et unique. Il faut bien voir cependant que l'accusation de Derrida s'autorise non pas de ce que le rapport de Heidegger

59. Heidegger, Approche de Hölderlin, p. 49.

60. «Notre pensée d'aujourd'hui a pour tâche de prendre ce qui a été pensé de façon grecque pour le penser d'une façon encore plus grecque. [...] S'engager à penser cet impensé, cela veut dire: entreprendre plus originalement ce qui a été pensé de façon grecque, le prendre en vue dans sa provenance» (Heidegger, Acheminement..., p. 125).

61. Collection Jacques Derrida. MS-C001. Boîte 12, dossier 12, feuillet 16 (séance 8). Special Collections and Archives, The UC Irvine Libraries, Irvine, California. Archives consultées en juillet-août 2018. Voir Derrida, La vie la mort, p. 222. 
au legs repose sur un présupposé, car il y a toujours du présupposé impliqué dans la lecture et dans l'héritage, mais des conséquences appauvrissantes de la mise en auvre de cette conception exclusive du legs, qui risque de forclore la possibilité d'un à-venir de l'héritage ${ }^{62}$.

Fatalement, il ne peut y avoir pour Heidegger qu'un nom propre, une signature, un lieu, un impensé et un Geschlecht. Et en vertu de cette pauvreté du legs, il se retrouve immanquablement seul sur la scène de l'héritage. Comme le note Derrida ${ }^{63}$, chez Heidegger la pensée est pauvre - doit être pauvre - parce qu'elle ne pense qu'une unique pensée. De même, c'est parce que la multiplicité éparse des poèmes de Trakl renvoient tous à un seul et unique Gedicht ${ }^{64}$ qu'ultimement ils sont riches et présentent le plus de valeur pour Heidegger. La force rassemblante - ce que dans un autre contexte Heidegger nomme l'esprit - semble régulièrement être chez lui l'étalon qui mesure le poids d'une pensée, d'un Gedicht ou d'une interprétation concurrente.

Contre Heidegger, ne pourrions-nous pas alors penser que ce n'est pas l'origine qui fait la richesse d'un legs, mais la tradition qui le porte, le développe et l'enrichit? Et qui a dit que la tradition à laquelle nous appartenons est bien une et exclusivement de source grecque? Faut-il en effet penser l'héritage comme Heidegger pense les deux coups du Geschlecht, c'est-àdire comme dispersion factuelle (tradition chrétienne et platonicienne, pensée et technique modernes, subjectivisme, etc.) d'une racine grecque impensée? Et quoi du legs judaïque, pour ne rien dire des apports chrétien, latin et arabe si on ne fait pas d'eux de simples «époques» de l'oubli de l'être, dans toute cette querelle de l'héritage ${ }^{65}$ ?

$$
* * *
$$

Le legs reste. Il serait bon d'en disposer enfin. C'est sans doute notre rêve à tous - et celui de Heidegger, jusqu'à l'obsession peut-être. Mais le rêve tourne invariablement au cauchemar: le legs ne se laisse pas approprier sans reste. Toujours une partie du legs (les jambes, peut-être) résiste, comme un corps encombrant que l'on a du mal à faire disparaître. Gênant, ce legs qui

62. C'est parce que l'héritage est livré sous la forme d'une promesse qu'il demeure irréductiblement à venir. Voir Jacques Derrida, Voyous, Paris, Galilée, 2003, p. 120 et Sauf le nom, Paris, Galilée, 1993, p. 108. Cette notion de promesse constitue l'un des lieux où le désaccord entre Derrida et Heidegger est le plus significatif. Elle oriente discrètement leurs conceptions respectives de l'héritage. Derrida met en scène ce désaccord dans De l'esprit, p. 144-154 et dans Mémoires. Pour Paul de Man, Paris, Galilée, 1988, p. 102.

63. Collection Jacques Derrida. MS-C001. Boîte 12, dossier 12, feuillet 15 (séance 8). Special Collections and Archives, The UC Irvine Libraries, Irvine, California. Archives consultées en juillet-août 2018. Voir Derrida, La vie la mort, p. 221.

64. Heidegger, Acheminement..., p. 43.

65. Je fais écho aux préoccupations de Marlène Zarader dans son Heidegger et les paroles de l'origine, p. 279-280, préoccupations développées dans La dette impensée. Heidegger et l'héritage hébraïque, Paris, Seuil, 1990. Voir aussi Derrida, De l'esprit, p. 164-167. 
ne se laisse pas enfermer (dans un cercueil, magnifique peut-être, mais terriblement clos) et mettre en terre - qui doesn't want to stand still - avec la sérénité (die Stille) que l'on reconnaît parfois au cadavre. D'où sans doute le soupir de Derrida: "Nous ne nous débarrasserons jamais du corps de Heidegger ${ }^{66}$.» Quelque tentation que l'on éprouve et quelque effort que l'on fasse, le legs reste parce qu'il ne reste pas en place et dans un lieu $(\ddot{O} r t)$ un. Le legs reste, dirait Derrida, parce que l'héritage est pluriel, d'une pluralité non polysémique mais disséminale.

Il faut reconnaître dans cette opposition entre polysémie et dissémination le lieu d'un chiasme. Dans Geschlecht III (p. 96 sq.), Derrida ne manque pas de faire porter l'insistance de son analyse sur cette opposition et ce qui en détermine la polarité chez Heidegger (bonne polysémie, mauvaise dissémination). J'ai indiqué pourquoi cette polarisation fait l'objet d'une évaluation négative - voire d'une accusation — de la part de Derrida. Pour ce dernier, la fécondité ou la productivité du legs ne peuvent faire l'économie de la dissémination. Celle-ci demeure toujours possible là où le sens est mis en jeu, envoyé, légué. Toute décision visant à l'exclure ou à la neutraliser pour assurer la pérennité du legs contredit sa propre intention.

Il ne faut cependant pas sous-estimer la nécessité de la détermination heideggérienne (rassemblée-rassemblante) du legs. La question du legs (legare) et de l'héritage peut être lue comme une manière de poser la question du lien (ligare) politique, et plus largement la question de la communauté (Geschlecht, encore). Comment, à quelles conditions peut-il y avoir communauté à travers la dispersion sinon en postulant (injonction pratique plus qu'hypothèse théorique) un lien qui nous ré-unit et nous rassemble? Pour Heidegger, ce lien est l'héritage, l'appartenance à une tradition et la correspondance avec elle. Nous aurions tort de sous-estimer la force de cette question en dépit des réticences de Derrida à l'endroit de la réponse heideggérienne, qui décrit selon Politiques de l'amitié une "schématique de la filiation ${ }^{67}$ ». Cette question demeure, encore aujourd'hui - surtout aujourd'hui —, d'une brûlante actualité. Sans trouver chez Heidegger, chez Blanchot, chez Nancy, chez Bataille et chez Lévinas, une réponse satisfaisante ${ }^{68}$, Derrida, comme nous tous et toutes, demeure pris avec elle: comment penser un rassemblement qui ne revient pas au même? Politiques de l'amitié représente à cet égard l'effort le plus explicite de Derrida pour affronter cette question, qu'il pense sous le titre de l'aimance. La réponse derridienne est-elle plus satisfaisante que celle de Heidegger ou reposent-elles tout simplement sur deux affects différents ou deux variantes (grossièrement: le cosmopolitisme

66. Voir supra, note 1.

67. Derrida, Politiques de l'amitié, p. 12-13, voir aussi p. 273.

68. Ibid., p. 97-100 et p. 331. 
et le nationalisme) de ce que J.-L. Nancy nomme l' "affect politique ${ }^{69}$ ? Je laisse la question ouverte.

«Et si, demande Derrida dans «Désistance», l'impensé de Heidegger $[\ldots]$ n'était pas un, mais pluriel ${ }^{70}$ ?»

\section{Pluraliser - le legs de Heidegger}

Cela pourra s'entendre au moins de deux manières. D'une part sous la forme d'une injonction: il faut pluraliser l'héritage heideggérien et pluraliser ce que Heidegger a dit de l'héritage, pour éviter que sa scène, aussi géniale soitelle par moments, ne se transforme en désert ou en soliloque. Mais d'autre part et par l'intermédiaire de Derrida, le legs de Heidegger aura aussi été de nous apprendre, contre toute attente et malgré lui, la vertu du pluralisme. Car ce qui ressort de toute cette scène d'héritage, où l'un cherche à hériter de l'autre, où l'autre cherche à hériter de l'un et de l'unique, c'est que le legs de Heidegger aura bien été pluriel, et pluralisé.

69. Voir Ginette Michaud, Cosa volante. Le désir des arts dans la pensée de J.-L. Nancy, Paris, Hermann, 2013, p. 147-148.

70. Derrida, «Désistance», Psyché, p. 616. 